\title{
APPROACH FOR A MACHINE-INTERPRETABLE PROVISION OF STANDARD CONTENTS USING WELDED CONSTRUCTIONS AS AN EXAMPLE
}

\author{
Manoharan, Thivakar; Loibl, André; Nagarajah, Arun; Köhler, Peter \\ Universität Duisburg-Essen
}

\begin{abstract}
In order to meet the quality standards required in today's product development process, the designer must be able to draw on the knowledge contained in standards at all times. However, in today's digital work environment, these are usually only available in paper or PDF form. To support the designer during the product development process, a research project examine how knowledge from standards can be made available digitally and integrated into his working environment. This paper presents a concept with a RESTful service as a central knowledge base, which provides knowledge in the form of microservices. The implementation is carried out using welding assemblies as an example. To achieve the high-quality requirements and to implement them, the standard contents had to be prepared in a machine-interpretable and cross system way.
\end{abstract}

Keywords: Knowledge management, Design practice, Information management, Case study

\section{Contact:}

Loibl, André

Universität Duisburg-Essen

Produktentstehungsprozesse und Datenmanagement

Germany

andre.loibl@uni-due.de 


\section{INTRODUCTION}

Quality demands made on various results, products and processes involved in the product development process require a high level of knowledge by all stakeholders involved. Standards are essential in the provision of knowledge. Because standards are administered by a superior authority and regulate international standards, they serve as a foundation in today's international and borderless trade. An important stakeholder of this knowledge is the designer. In the initial phase, the designer lays foundations for products conforming to standards. Therefore, he is obliged to have an experienced handling of standards.

Due to his interdisciplinary work, he has a special responsibility. Because he carries expertise from different areas together and has to implement it accordingly as well as considering the consequences of his decisions. In order to this and to be legally protected, a large part of his work consists of searching through standards and extracting knowledge relevant to him. These standards are usually available in paper form or as PDF files.

Both formats have in common that filtering out required knowledge is very time-consuming, because standard documents are not context-specific. In addition, the documents still must be processed manually, which is very time-consuming and a frequent source of errors. Therefore, there are already service providers who offer implementation of standards in IT systems, such as calculation programs. These are mostly isolated solutions that do not offer interfaces to CAD or PLM systems. Here, too, the results are transferred manually, which results in a break in information and is highly susceptible to errors, leading to a concrete endangerment of quality requirements.

To support the designer, the concept of a central knowledge base was developed and implemented as part of a research project. For this purpose, the contents of standards are made firstly machine interpretable and secondly cross-system to meet the high-quality requirements and to implement them. To achieve this goal, the contents of standards must first be prepared in a way that can be interpreted by machines. The knowledge base then links the most diverse sources of knowledge, such as calculation software, CAD systems and standard knowledge. The knowledge from the knowledge base is made available and managed by modern information technologies. So that also a necessary documentation of the individual process steps is possible.

\section{STATE OF THE ART}

Two problems were identified in the introduction. The first one is that standards are not machine interpretable and the second one that they are not made available across systems. In the following, the current solution of these two inadequacies will be shown.

Today, machine interpretability is solved by users searching through the standard manually and applying the knowledge relevant to their work. Ideally, a documentation about the founded standard content should be provided, so it is immediately available for subsequent access. This activity is also offered by service providers when a use case is detected. A typical example of those calculation programs are for calculating pressure vessels, like espresso from MDesign. Calculation software manufacturers have converted the calculation specifications described in the corresponding standards into program code and make them available with an attractive user interface. A further example are standard parts catalogues, some of them have already been implemented into CAD-systems by CAD manufacturers, so that there is no need to redesign screws.

Another method for extracting knowledge from standards is the usage of Knowledge-Based Engineering (KBE). A general procedure of creating a KBE system is contained in VDI 5610 Part 2 (2017). As Kesselmans has already stated, the support of the designer can only take place if mechanisms are provided with which it is possible to process construction knowledge (Kesselmans, 2014). For this purpose, a platform must be made available in which this knowledge is stored: a knowledge base. Knowledge acquisition and the creation of the knowledge base are the basis of every KBE method. The Knowledge must be extracted and structured out of Norms. Techniques for acquiring knowledge, both explicit and implicit, can be found in different sources such as (VDI 5610 Part 2,2017).

Further activities which are also done by official authorities regarding machine interpretability of standards are, for example, the fact that the trend towards digital content services has been taken up by DIN Software GmbH. Concepts are currently being considered and some are already being offered, such as how to make standard contents available in XML format and thus make them available in various output formats. The standard of the US law NISO will be oriented towards a standard of NISO which has 
published a standard for a format of journals ("ANSI/NISO Z39.96-2012 JATS: Journal Article Tag Suits") which corresponds to the needs of ISO (Wischhöfer, 2016). In addition, new interfaces will be offered which can be used by external applications to provide full texts of standards and metadata digitally for platforms, customers and external partners. This will be realized by a so-called extensible semantic content repository (Koch et al., 2017). However, as already mentioned, the focus of this paper is the conversion of the full texts of standards into a XML format and provision of standard content.

Current solutions for making knowledge available across systems are usually local linking of different programs. To make this possible, software manufacturers must provide an application programming interface (API) so that the functions of the respective software can be accessed. Such APIs are contained in every larger CAD system and thus enable the integration of external programs like in Siemens NX, PTC Creo or Autodesk Inventor.

Another way to get access to external data is in many current contributions, the access to external data via the CAD-system is web-based. The papers of Danjou and Manoharan are noteworthy upon using the feature technology focused on variant constructions. Danjou et al. (2005) present the use of UDFs exemplified by an automated compressor configuration. The necessary parameters are stored in a database, and a UDF library is created for the individual UDFs. Depending on the requirements, a configurator places the UDFs in the CAD assembly. Moreover Manoharan et al. (2016) shows knowledge integration in the CAD-CAM process chain through the example of $\mathrm{NC}$ and additive manufacturing. Here, the author implements a web service for linking the CAD system with the relevant databases (Fig. 1).

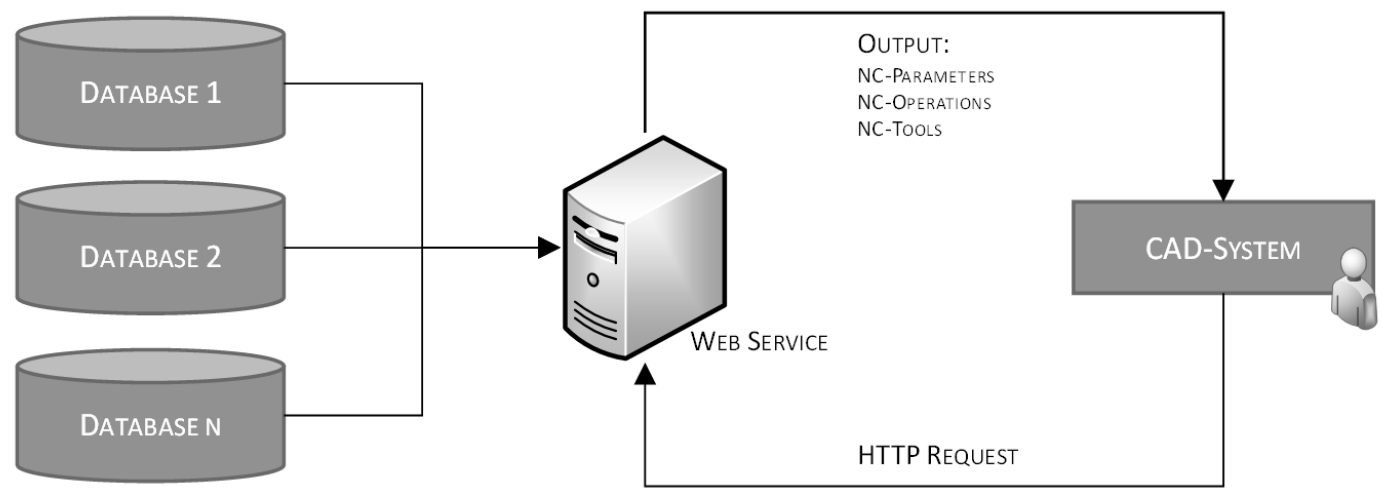

Figure 1. Linkage databases - web service - CAD-system (Manoharan et al., 2016)

According to Manoharan et al. (2016), one important advantage for using a web service is if changes are needed in the database structure. This ensures that only the web service or the database has to be adapted and not the expert system.

In addition to the two deficits described, the designer today is confronted with interdisciplinary tasks and is required to have knowledge in many fields. To support these requirements, the following case study shows how knowledge from standards can be made available digitally, i.e. how knowledge can be interpreted by machines and how it can be prepared context-specifically. Welding constructions will be used as an example.

\section{APPROACH FOR A CENTRAL KNOWLEDGE BASE}

At first data and information are made available to the designer that previously had to be searched for or determined manually. For example, this includes data and information on the welding process from welding software, calculation results from container design software or standard contents and standard documents.

The entire concept is based on a central knowledge base that provides the knowledge in form of services. These services can be published via the Internet or a company-owned intranet. Communication with the services takes place via HTTP protocol. Only a defined interface (API) for communication must be defined. These services access databases and software systems and contain the logic for linking and using the data. Thus, different clients can access the same database without direct integration of databases or connecting of software systems. A structural change of the database or even the exchange of a software system can take place without adaptation of the client as long as the defined API is adhered 
to. Figure 2 shows the concept of the project "Knowledge-based CAx process chains for welded structures in power plant construction"(WPSK) funded by the Federal Ministry of Economics and Energy.

The use of information can be divided into two phases, the data entry phase and the implementation phase. The basic differences are that in the acquisition phase information must be manually extracted from the standards and converted into a machine-processable format. Thus, the data from standards and regulations were digitally converted into the Json-format. In the first instance, only quantitative information from standards, such as formulas or tables, was transferred.

In the implementation phase, the previously transferred data is automatically made available by the service. The data is also made available in neutral Json-format, which enables further use in the CAD system, independent of which service was called.

The service for providing the knowledge base was implemented by the project partner CAD Schroer $\mathrm{GmbH}$ and published for all project partners. On the server side, the services are created with Spring Boot and the necessary infrastructure is built with Spring Cloud (Wolf and Flohre, 2015). Hereby several small services (Microservices) were created, each of them performs a certain small task in a defined context. These microservices run in a Docker environment (Vohra, 2016). The communication with the microservices and with each other is only done via REST (Representational State Transfer) (Fielding, 2000). Each request URL contains the information needed to process the request. In most cases, the response is returned in JSON format.

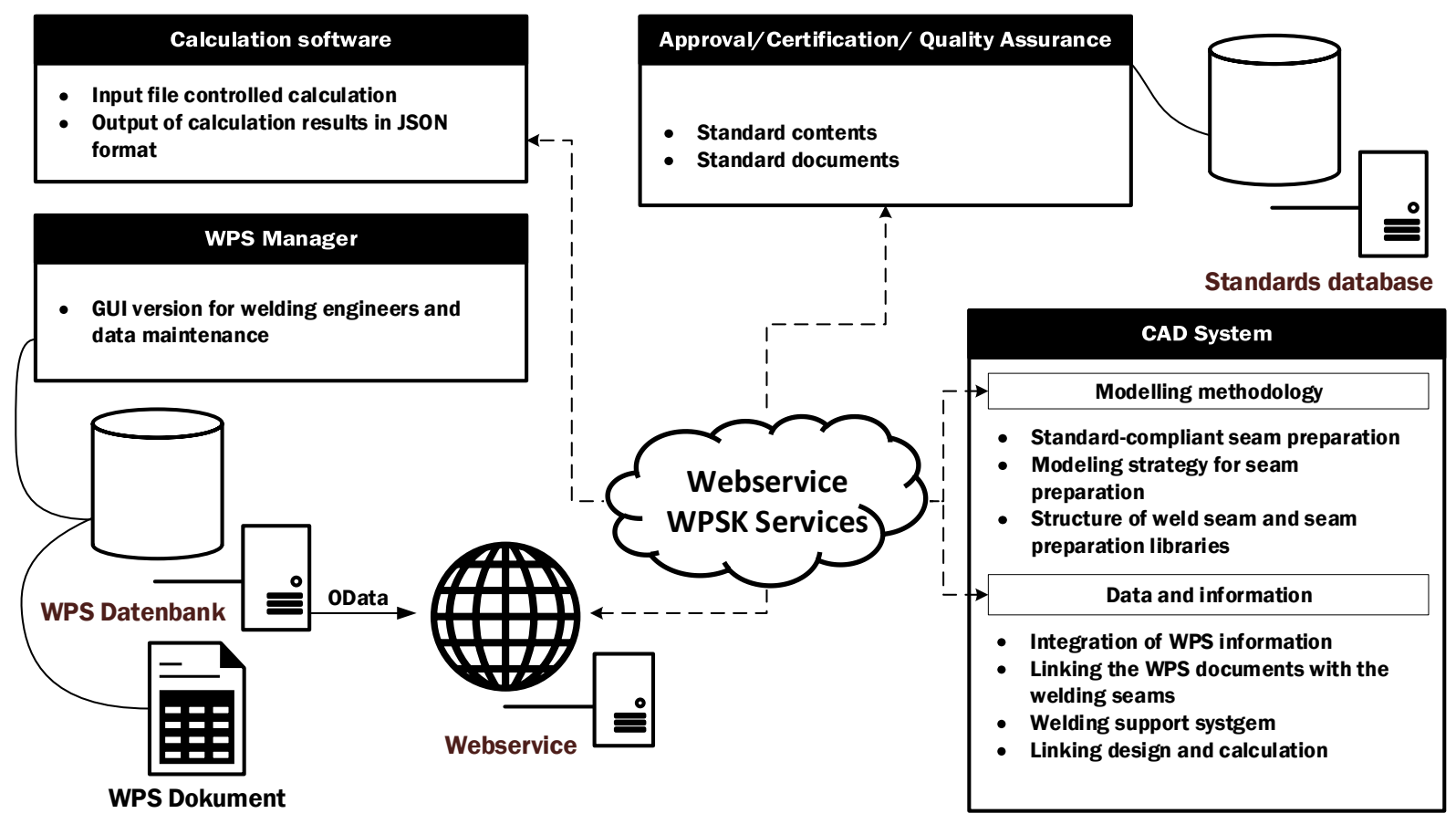

Figure 2. Concept of a central knowledge base

Figure 3 shows the structure of the Microservices (WPSK Services). In summary, it can be said that the Config Service provides the configuration settings for all other services and these can be retrieved. The Discovery Service registers all services so that the Edge Service can request which service is available at which address and which requests are being processed. The detailed functionality of the Config Service and Discovery Service is not discussed in this article. Further explanations can be found under (Rv, 2016). Different clients can access the services platform independent using HTTP requests if the WPSK API is observed. These requests are first forwarded to the Edge Service which contains a Microservices API Gateway, a proxy that enables the clients to use the WPSK API functionalities. The API was defined by a cross-functional team and is a REST based interface that exchanges data in JSON format. Once an API has been defined, both the server application and the client application can be developed independently. Thus the incoming requests, defined by the WPSK API, are forwarded to a service behind the gateway. If one of the microservices requires the functionality of another service, it also communicates via the edge service. The concept includes 
several load balancers, which start one or more additional instances of the microservices depending on the utilization of the individual services and thus process the requests in a distributed manner.

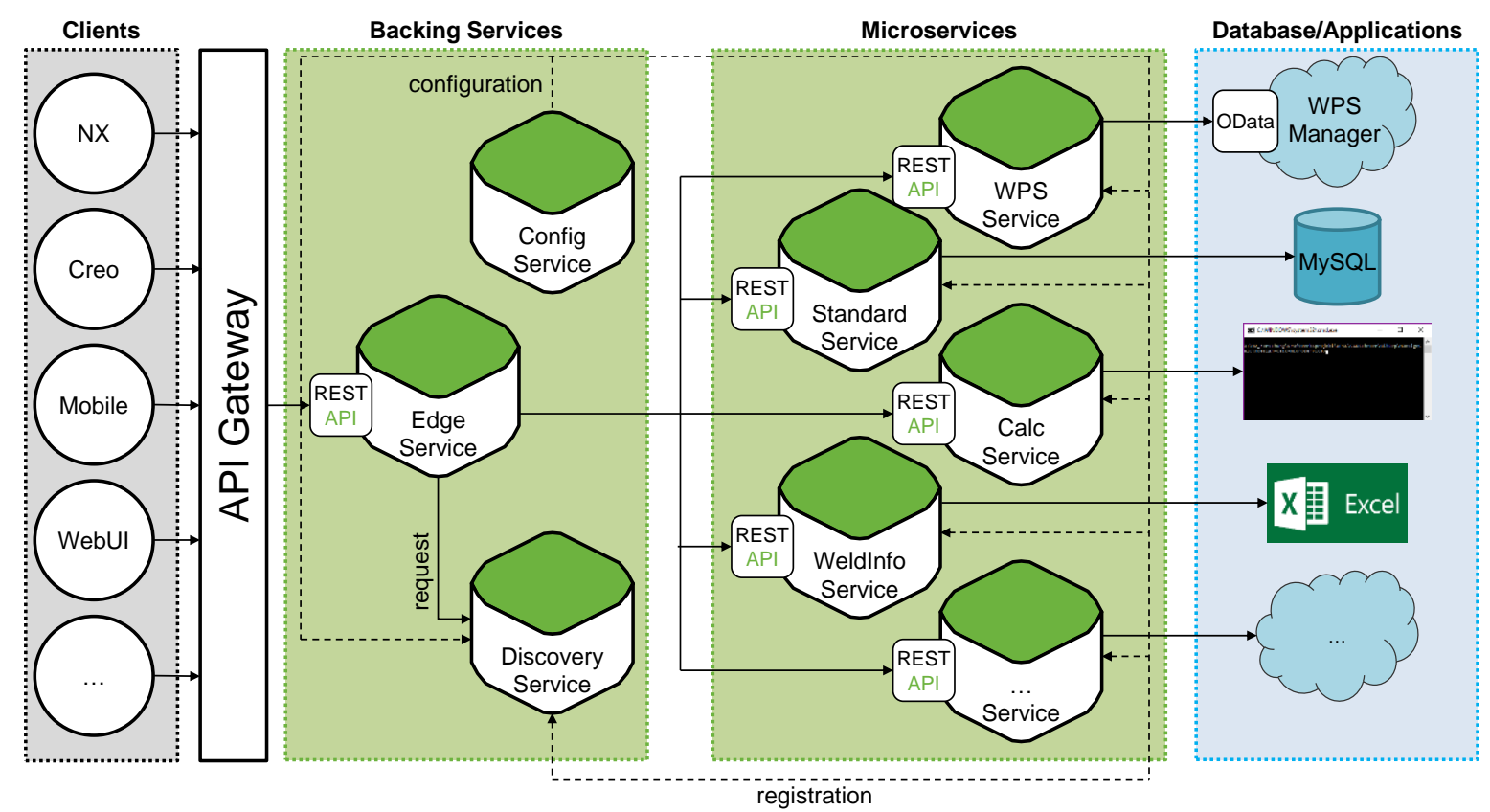

Figure 3. Structure of microservices

Microservices are small, decoupled services that can in turn access other services, databases or software tools. The Microservices can be easily and quickly added to functions and even exchanged. If the externally defined interface is not changed, the client will not notice anything of an exchange. For example, you could switch to another calculation software or use two different software solutions in parallel for different queries. In the following some example services and the integration of these into the construction process are explained.

\section{CASE STUDY: INTEGRATION OF MICROSERVICES IN THE DESIGN PROCESS}

The following chapter will show you how microservices, which contain knowledge prepared machineinterpretable, can be integrated in the design process. Three examples are shown one for implement a calculation service, one for validating weld seams and the last one offers information's and data from standards.

\subsection{Calc service}

Especially in the field of pressure vessel design, the linking of design, calculation and documentation is indispensable. To maintain a uniform data-consistent model and to provide all the documents required for a comprehensive product life cycle, the designer is increasingly required to perform initial calculations while designing. Many identical data required for these steps are available in different models and must always be transferred manually. Thus, the 3D model and the calculation model are usually separated from each other. This problem was investigated in (Kesselmans, 2014). However, the models and interfaces developed there require manual adjustments.

These two worlds currently still exist in parallel for most part without any link or possibility of data exchange. One consequence of this state is that a high number of iteration steps are required from the designer in the development phase until he achieves the desired result. These iteration steps contain a high error potential. Thus the designer can exchange or incorrectly transfer the parameters in both directions during the transfer of the parameters. In addition, if there is insufficient specialist knowledge or if a parameter is changed, the assumption can be wrongly made that a new calculation is not necessary. Furthermore, a high number of iterative and repetitive activities lead to a high expenditure of time and therefore represent a not inconsiderable cost factor. 
This leads to the fact that it is necessary to link these two worlds. The designer is relieved and can concentrate on his actual tasks, e.g. validating calculation results. Or he can deal more intensively with more complex interrelationships and can thus develop higher-value solutions. More, calculation dependencies that are standard-wide can also be mapped, so that the designer is directly shown information about which calculations still must be carried out if a parameter is changed.

The calculation integration takes place based on national and international rules and regulations. These include the codes and standards AD 2000 Merkblatt B 0 (AD 2000-Merkblatt B 0, 2014), DIN EN 134453 (DIN EN 13445, 2016) or ASME BPVC Section VIII, Division 1 (ASME Boiler and Pressure Vessel Code). Software solutions available on the market, usually provide a comprehensive selection of regulations. Within the scope of this project, an exemplary implementation of DIN EN 13445 is carried out. The results can be easily transferred to other standards with the aid of minor adaptations.

Another challenge is to ensure cross-component compliance with the rules and regulations. The aim is to ensure that interactions between stress and weld design are considered. So correlations are maintained and updated accordingly in the event of changes.

The tool espresso from MDESIGN was selected, espresso provides calculation modules for pressure vessels according to AD2000 and DIN EN 13445. It is possible to carry out calculations in batch mode. In this mode, MDESIGN XML files are called up in which the components, load conditions and material properties are described. The results are also returned in XML format. To guarantee a calculation documentation a PDF document is created.

The tool is integrated and used by a micro service. The URL that is called is as follows:

\section{http://[WPSKServicesDomain]/api/v1/calc/pressureTank/shell?type=cylindrical\&de=1600\& $d i=1560 \&$ en $=20 \& l=4000 \&$ material $=20 \mathrm{CrMoV} 13-5-5 \&$ materialnr $=1.7779 \& p a=9 \& t=100$}

Table 1. Query parameter Calc service
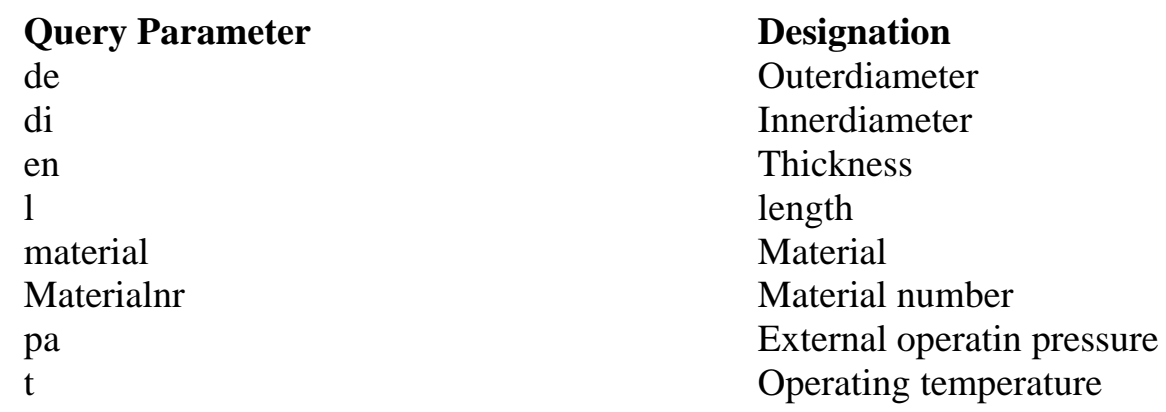

By specifying these parameters, calculation is carried out and existing stress (fa) and maximum permissible pressure (pi_max) are returned as results. The results are returned to the client in JSON format after the XML output file has been converted, which is returned from MDESIGN. A link to the calculation documentation in PDF format is also generated.

Figure 4 shows the data and information flow during the calculation integration. This is based on a link between CAD software and a calculation program. To realize this linking, adjustments must be made in the CAD model. Additional parameters must be stored which are relevant for the calculation but have no significance for the CAD model, e.g. external operating pressure and operating temperature. This is checked by the KBE application and only after a positive feedback, the Microservice can be started. 


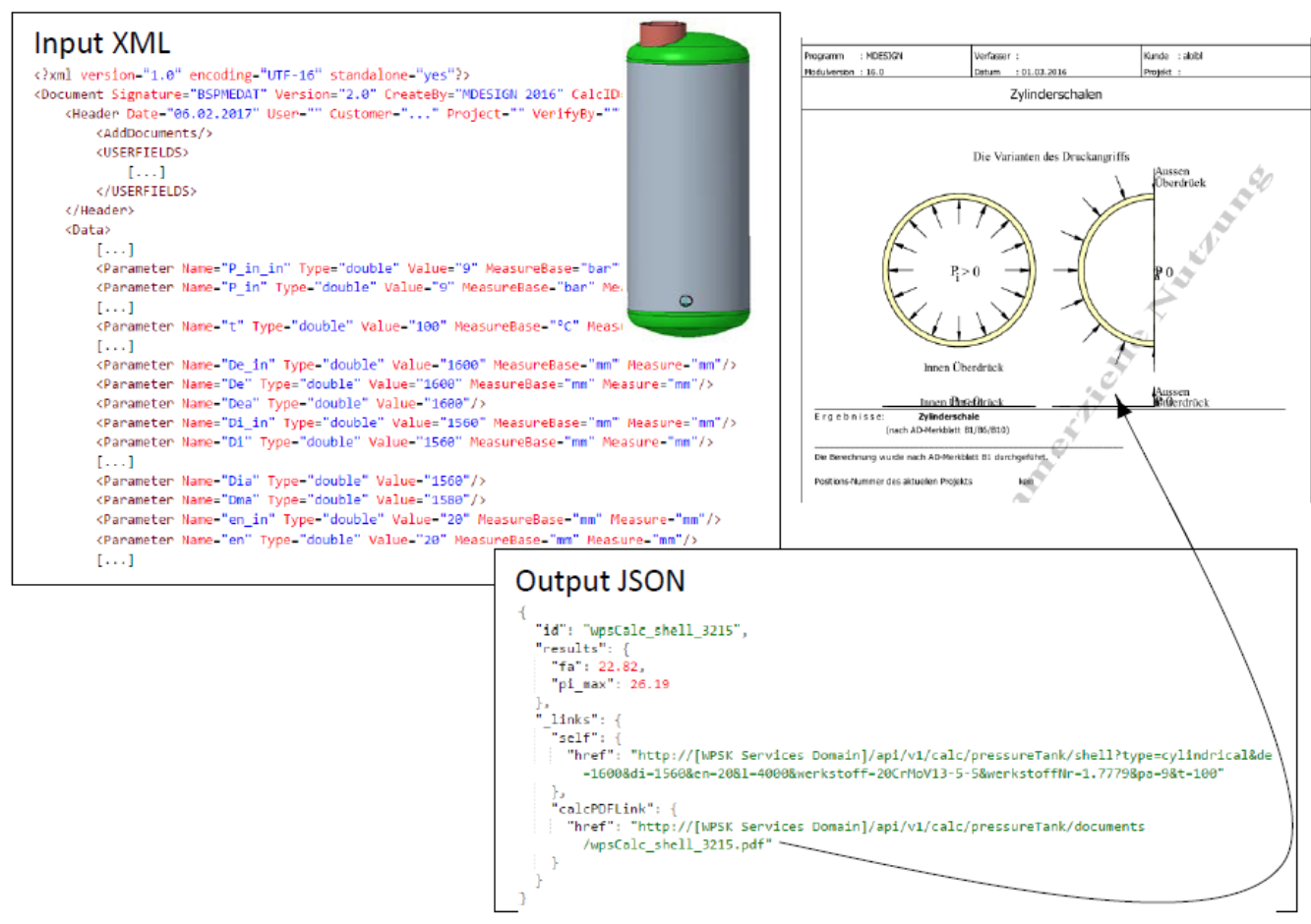

Figure 4. Process of calculation integration

After returning the calculated values and the link, a corresponding parameter is created in the CAD model and saved. So a uniform and consistent database is available.

Also, a model-based calculation model is being added which stores dependencies between the individual components of the assembly and provides the designer with corresponding information. Also design rules are implemented, regarding to positioning of nozzles and associated cut-outs, so they are secured by standards.

\subsection{WPS service}

This service offers the client the possibility to retrieve data from a welding software. In this case it is the so called WPS Manager of the project partner SLV Halle. In addition to the GUI version, the WPS Manager offers a web service where data can be retrieved from the database. The Open Data Protocol (OData) (odata, 2017) is used for this purpose. Thus, by using the OData URI Conventions (Cheng, 2012), the entire database content can be retrieved without each resource having to be defined individually. For example, it is possible to search for a suitable WPS or WPQR for a given welding task. Or the data of a certain WPS or WPQR. The service also offers a functionality to retrieve the corresponding PDF document.

To select a suitable WPS or WPQR, the semi-finished products must be defined, this can be a tube (T) or a sheet metal $(\mathrm{P})$, where a tube with $\mathrm{D}>500 \mathrm{~mm}$ is regarded as a sheet metal. In addition, the material of the semi-finished products, the wall thicknesses, the weld shape (fillet weld - FW or butt weld - BW) and the weld thickness are required. In the case of a pipe, outer diameter is also required. Table 2 shows the difference of the request URLs between the WPS Manager Webservice and WPS Services. The long URL of the WPS Manager Webservice (left column) is due to the database structure and the OData interface. By defining an API for this problem, the URL in the right column can be called. 


\section{Table 2. Comparison of request URLs}

\section{WPS Manager Webservice Request URL}

http://[WPSManagerWebserviceDomain]/wpsserv ice/wpsdata/weldinginstructionbase/weldinginstru ction? \$format=JSON\& \$expand=WSGroup,seamt ype,SheetTube,Processs\& $\$$ filter=SheetTube/semif inishedproductabbrevationeg ' $\mathrm{T} / \mathrm{T}$ ' andworkpieceth icknesseg12andouterdiametereg762andworkpiecet hickness2eg12andouterdiameter2eg340andmateria lgroup/Numbereg '8.2' andseamtype/eg 'BW'\&\$sel ect=process/proceedingnr,process/process, materia lgroup/shortdescription,materialgroup/description, materialgroup/number,seamtype/seamtypeshort,se amtype/seamtypedescription,sheetTube/semifinsh edproductabbrevation,sheettube/semifinishedprod uctdescription,created,WPSnumber,workpiecethic kness, workpiecethickness2,outerdiameter,outerdia meter2,seamlength,filletthickness

\section{WPSK Services Request URL}

http://[WPSKServiceDomain]/api/v1/wpsm/wp ss/search?semifinishedproductabbrevation $=\mathrm{T} / \mathrm{T}$ $\%$ workpiecethickness $=12$ outerdiameter $=762 \&$ workpiecethickness $2=12$ \&outerdiameter $2=340$ \&materialnr=8.2\&seamtypeshort $=\mathrm{BW}$

The WPSK service can return the response adapted to the defined API.

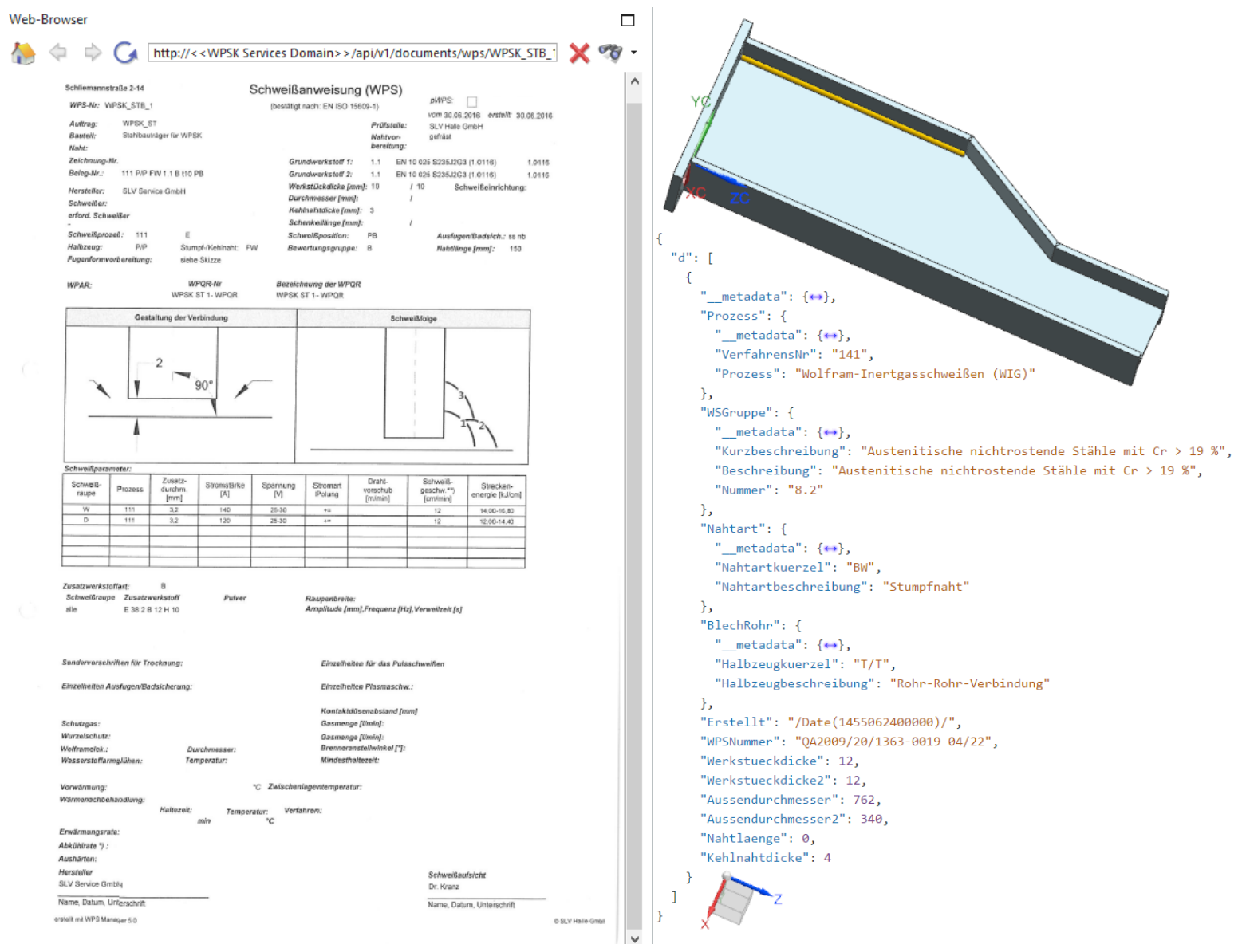

Figure 5. NX Client for WPS Service

Figure 5 shows the implementation in NX. When a weld feature is placed, the data required for the query is read from the selected parts and sent to the WPSK service. The JSON response file contains all the information needed to generate the weld geometry, fill it and the weld process feature with the appropriate data. When the weld feature is created, a link to the WPQR and WPS document is saved in the $3 \mathrm{D}$ note so the user can review it at any time. 


\subsection{Standards service}

The Standards Service offers the designer data and information from the standards and guidelines. Here, too, suitable APIs have been defined depending on the requirements on the client side, so that the designer always receives the necessary data. The WPSK Service can also display the PDF documents of the standards here. Currently, only selected parts of standards have been implemented, e.g.: Tables, formulas, graphs and illustrations. The reason for this is the current way in which standards are provided. Standards are available digitally, but only in the form of PDF documents. In order for computers to be able to process these data further, the standard must first be prepared manually. The provision of standards in the form of PDF documents must be reconsidered in future. Within the framework of this project, possibilities are currently being investigated for making standards available in the form of document-based databases and for generating a PDF file from these on request.

\section{DISCUSSION}

During the implementation of the central knowledge base, within the framework of the project, many findings were collected. On the one hand many discussions with experts in the respective fields showed that we are far from developing fully automatic systems. Which automatically extract the knowledge from a standard and make it available to the user in a context-specific form. There is still a high manual initial effort necessary to prepare the data and to decide which standard is worth the effort. Here a classification system would be helpful to estimate in which description form (formal, semi-formal or abstract) a guideline or standard exists. As a previous step it has to be define, what formal, semi-formal or abstract means.

So all in all today and in the future, there will always be the dependency on the knowledge of experts and we will not be able to replace them.

The implementation of the knowledge base with microservices in to the product development process proved as a good solution. All services can be addressed via the specially developed API, whereby the queries have the same syntax. A further advantage is that exchange of software can take place without any problems, the user will not recognize anything. It can be done while the system is operating. Because behind a Microservice different programs can work, the user uses for all cases an identical query. However, it can also be said that it will be continued in the future that a direct coupling between software systems is necessary and knowledge will be stored in different systems, depending on application and where it is intelligently linked.

Regarding to standards, the following conclusions can be drawn: there is an urgent need for action to define an international standard in order to make standards machine-readable and machineinterpretable. In large standards, such as DIN EN 13445, it is very difficult to extract specific knowledge for one's task if there is no context-specific provision of knowledge. For the contextspecific provision of standard content, it is essential that the knowledge is classified.

\section{OUTLOOK SUMMARY}

The article shows the concept of a central knowledge base which makes knowledge available to the designer digitally and context-specifically. Subsequently, implementations were shown how standard contents can be made available in a machine-interpretable and cross-system manner - using microservices. The developed concept was implemented using welded constructions as an example, as this was the focus of the research project. During the development of the approach, care was taken to ensure that it was generally valid, with the restriction that the standards had to be available in a high formal format. For the application of the approach to standards such as DIN EN 9001, further questions have to be clarified. So it can easily be transferred to other standards which mainly describe information qualitative. Like for standards for bolts, feather keys or bearings.

However, it can be stated that, as state of the art, a broad application of this method is too costly to achieve, new research focuses can be derived. It is necessary to investigate how standards must be made available in the future so that they can be used in the context of Industry 4.0. Essential questions arise:

- what are suitable forms of representation,

- which standards are practicable for digitisation and

- how to classify knowledge contained in standards to define criteria for preparation of individual contents. 
Another question that needs to be answered is how different standard versions can be managed in the Industry 4.0 environment. Products or projects are manufactured and produced according to the standard valid at the time of the project; today these valid statuses can be viewed at any time in PDF and paper form or are managed by a standard management systems. However, if context-specific standard contents are now offered for systems and machines in the Industry 4.0 environment, it must be ensured that systems and machines know which version of the standard is valid or has been used for the respective product. It must therefore be ensured that different versions of the standard can be accessed at any time and that they are assigned accordingly. A classification methodology must also be developed here.

\section{REFERENCES}

AD 2000-Merkblatt B0. (2014), Berechnung von Druckbehältern

ASME B. and Pressure Vessel C. "ANSI/ASME BPV-VIII-Z Section VIII Rules for Construction of Pressure Vessels". Devision 1

Cheng, S. (2012), "OData programming cookbook for NET developers". 70 fast-track, example-driven recipes with clear instructions and details for OData programming with NET framework, Packt Pub, Birmingham.

Danjou, S., Lupa, N. and Köhler, P. (2008), "Approach for Automated Product Modeling Using KnowledgeBased Design Features", Computer-Aided Design and Applications Vol. 5, 2008. http://dx.doi.org/10.3722/cadaps.2008.622-629

DIN EN 13445-3 (2016), Unbefeuerte Druckbehälter - Teil 3: Konstruktion, Deutsche Fassung EN 13445-3: 2014, Beuth Verlag, Berlin.

Fielding, R. (2000), "Architectural Styles and the Design of Network-based Software Architecures", Ph.D. Thesis, University of California.

Kesselmans, C. (2014), "Höherwertige Konstruktionsobjekte für CAD-Prozesse", Ph.D. Thesis, University of Duisburg-Essen, Duisburg.

Koch, H., Willuhn, H. and Esser, M. (2017), "Digitale Content-Dienstleistungen aus dem zentralen XML Content Repository", DIN Mitteilungen, Beuth Verlag, Berlin.

Manoharan, T., Humpa, M., Martha, A. and Köhler, P. (2016), "Knowledge integration in CAD-CAM process chain", In Computer-Aided Design and Applications.

OData (2017), Avaiable at: http://www.odata.org/ (08.06.2017).

Rv, R. (2016), "Spring Microservices", Build scalable microservices with Spring Docker and Mesos.

VDI 5610 Part 2. (2017), "Knowledge management for engineering - Knowledge based engineering (KBE)", Beuth Verlag, Berlin.

Vohra, D. (2016), "Pro Docker", Learn how to use containers as a service for development and deployment.

Wischhöfer, C. (2016), "Standardisierung des ISO-XML-Formats für Normen”, DIN Mitteilungen, Beuth Verlag, Berlin.

Wolf, E, Flohre, T. (2015), "Spring Boot und Spring Cloud”. In Entwickler.press, s.l.

\section{Supported by:}

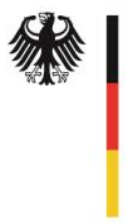

Federal Ministry

for Economic Affairs

and Energy

on the basis of a decision

by the German Bundestag 